\title{
miR-124 inhibits proliferation and invasion of human retinoblastoma cells by targeting STAT3
}

\author{
SHU LIU ${ }^{1 *}$, CHUNMEI HU ${ }^{2 *}$, YINGXUE WANG ${ }^{3}$, GUANG SHI ${ }^{2},{\text { YARONG } \mathrm{LI}^{2} \text { and HUANG WU }}^{1}$ \\ Departments of ${ }^{1}$ Ophthalmology, ${ }^{2}$ Tumor and Hematology, and ${ }^{3}$ Electrical Diagnosis, \\ The Second Hospital of Jilin University, Nanguan, Changchun, Jilin 130041, P.R. China
}

Received March 27, 2016; Accepted July 22, 2016

DOI: 10.3892/or.2016.4999

\begin{abstract}
A growing body of evidence suggests that microRNA-124 (miR-124) functions as tumor-suppressor, and involves in tumor initiation, development and metastasis in major classes of human cancers; however, the biological role and underlying molecular mechanism of miR-124 in retinoblastoma (RB) remain unknown. Therefore, we investigated the biological activity and underlying molecular mechanism of miR-124 in human retinoblastoma. In the present study, our results demonstrated the downregulation of miR-124 in $\mathrm{RB}$ tissues and RB cell lines compared with normal retinal tissues. The ectopic expression of miR-124 in the RB cell lines (Y79 and SO-RB50) suppresses cell proliferation, migration and invasion, induced cell apoptosis in vitro. Furthermore signal transducer and activator of transcription 3 (STAT3) was identified as a new target of miR-124, and overexpression of miR-124 decreased STAT3 expression on mRNA level and protein level in human RB cells. We also found that STAT3 mRNA expression was upregulated and inversely correlated with miR-124 expression in the RB tissues ( $r=-0.683 ; \mathrm{P}<0.001)$. Restoration of the expression of STAT3 rescues the effects induced by miR-124 in RB cells. The findings of the present study suggested that miR-124 functioned as tumor suppressor in RB, at least in part, by targeting STAT3, and that it could serve as a potential candidate for RB therapeutics.
\end{abstract}

Correspondence to: Dr Yarong Li, Department of Tumor and Hematology, The Second Hospital of Jilin University, 218 Zhiqiang Street, Nanguan, Changchun, Jilin 130041, P.R. China

E-mail: doctor.lyr@163.com

Dr Huang Wu, Department of Ophthalmology, The Second Hospital of Jilin University, Nanguan, Changchun, Jilin 130041, P.R. China E-mail:wuhuang728@sina.com

*Contributed equally

Key words: retinoblastoma, microRNAs, miR-124, STAT3, proliferation

\section{Introduction}

Retinoblastoma (RB), a deadly pediatric eye cancer, is the most common primary intraocular malignancy in children worldwide (1). The mortality rate among children with RB is $50-70 \%$ in the underdeveloped countries (2). The reason of high mortality rate of $\mathrm{RB}$ is mainly frequent metastasis of $\mathrm{RB}$ and intracranial neuroblastic malignancy (trilateral RB) $(3,4)$. It is an urgent need to further study the biology and molecular mechanisms of RB that cause RB procession and metastasis, and identify the specific biomarkers and therapy agents for improving the therapeutic outcome of patients with RB.

MicroRNAs (miRNAs) are a novel class of short (18-25 nucleotides in length) noncoding RNAs that regulate gene expression by repressing translation and cleaving their target mRNAs through binding to complementary sites in their $3^{\prime}$ untranslated region (3'UTR) $(5,6)$. It has been demonstrated due to aberrant expression. An accumulating body of evidence showed that miRNAs are involved in various biological processes such as development, differentiation, invasion, proliferation, apoptosis and stress response (7). It has been demonstrated that the altered expression of miRNAs contributes to the initiation and progression of cancer, and functions as tumor suppressors and oncogene $(8,9)$. Numerous miRNAs have been found to be involved in the development of RB $(10,11)$, suggesting that miRNAs may potentially serve as a novel strategy of diagnosis and therapy to RBs.

There is a growing interest particularly toward microRNA-124 (miR-124) in context of various types of cancers. miR-124 has been reported to be downregulated and function as a tumor suppressor in a variety of human cancers, such as prostate cancer (12), glioma (13), lung adenocarcinoma (14), breast (15), gastric (16) and colorectal cancer (17). However, the expression of miR-124 in patients with RB, and its biological functions in human RB cells, as well as the molecular mechanisms by which miR-124 exerts its functions, remains largely unclear. Therefore, the aims of the present study were to investigate the miR-124 expression in RB tissues and cell lines, and to evaluate its role and underlying mechanisms in RB. The result of the present study showed that miR-124 expression was downregulated in RB tissues and cell lines; and that overexpression of miR-124 in RB cells inhibited cell proliferation, migration and invasion and induced cell apoptosis in vitro by targeting signal transducer and activator 
of transcription 3 (STAT3). These findings provide a novel therapeutic strategy for treatment of RB.

\section{Materials and methods}

Human tissue samples and cell lines. Forty human RB and 20 normal retina tissues were provided by the First Hospital of Jilin University (Changchun, China). All tissue samples were harvested at surgery, immediately frozen in liquid nitrogen and stored at $-80^{\circ} \mathrm{C}$ until RNA extraction. The present study was approved by the Ethics Committee of the First Hospital of Jilin University (Changchun, China). All of the experiments were undertaken with the understanding and written consent of each patients or family.

Two human RB cell lines (Y79 and SO-RB50) were purchased from the Type Culture Collection of the Chinese Academy of Sciences (Shanghai, China), and were grown in RPMI-1640 medium (Gibco, Grand Island, NY, USA) supplemented with $10 \%$ heat-inactivated fetal bovine serum (FBS; HyClone, Logan, UT, USA), $100 \mathrm{U} / \mathrm{ml}$ penicillin or $100 \mathrm{mg} / \mathrm{ml}$ streptomycin in a humidified atmosphere of $95 \%$ air and $5 \%$ $\mathrm{CO}_{2}$ at $37^{\circ} \mathrm{C}$.

Transfection experiments. miR-124 mimic (miR-124) and corresponding miRNA-negative control (miR-NC) were purchased form GenePharma Co., Ltd. (Shanghai, China). The STAT3 sequence was amplified from human genomic DNA using a standard PCR protocol, and inserted into the pcDNA3.1 (Invitrogen, Shanghai, China). For the transfection experiments, $2 \times 10^{5} \mathrm{RB}$ cells were seeded in a $6-\mathrm{cm}$ dish in antibiotic-free RPMI-1640 medium with $10 \%$ FBS. Twenty-four hours later, the specific molecular production were transfected into the RB cells using Lipofectamine 2000 (Invitrogen, Carlsbad, CA, USA) according to the manufacturer's instructions.

Quantitative reverse transcription polymerase chain reaction (qRT-PCR). Total RNA from the cultured cells and frozen tissues was isolated using TRIzol reagent (Invitrogen) following the manufacturer's instructions. To quantify miR-124, total RNA was reversely transcribed into cDNA using One Step PrimeScript miRNA cDNA Synthesis kit (Qiagen, Valencia, CA, USA). Then, miR-124 level was detected using the TaqMan miRNA assay kits under ABI 7900 Fast system using primes of miR-124 and U6 (all from Applied Biosystems, Foster City, CA, USA). To quantify STAT3, cDNA was synthesized using PrimeScript RT reagent kit (Takara, Dalian, China). Then, STAT3 mRNA level was detected using the Real-Time PCR Mixture Reagent (Takara) under an ABI 7900 Real-Time PCR system. The primer of STAT3 and GAPDH was used as previously described (18). Relative expression of miRNA and mRNA was calculated using the $2^{-\Delta \Delta \mathrm{Ct}}$ method following normalization to U6 or GAPDH expression, respectively.

Cell proliferation and apoptosis analyses. Cell proliferation was determined using 3-(4,5-dimethylthiazol-2-yl)2,5-diphenyltetrazolium bromide (MTT; Sigma, St. Louis, MO, USA) assays. Briefly, $5 \times 10^{3}$ transfected cells/well were seeded into a 96-well plate and cultured for $72 \mathrm{~h}$. Then, $20 \mu \mathrm{l} \mathrm{MTT}$ solution $(5 \mathrm{mg} / \mathrm{ml})$ was added into each well and additionally cultured for $4 \mathrm{~h}$, then, MTT solution was removed and $150 \mu \mathrm{l}$ dimethyl sulfoxide (DMSO; Sigma-Aldrich, St. Louis, MO, USA) was added to each well. Optical density (OD) was detected at the wavelength of $570 \mathrm{~nm}$ using a Benchmark Plus $^{\text {TM }}$ microplate spectrometer (Bio-Rad, Hercules, CA, USA).

For cell apoptosis, the cells were collected $48 \mathrm{~h}$ after transfection and washed with phosphate-buffered saline (PBS). Then, $5 \times 10^{4}$ cells were resuspended in $500 \mu$ of binding buffer containing $5 \mu \mathrm{l}$ of Annexin V-fluorescein isothiocyanate (FITC) and $5 \mu \mathrm{l}$ of propidium iodide (PI), following the manufacturer's instructions of the Annexin V-FITC apoptosis detection kit (KeyGen, Shanghai, China). After incubation for $15 \mathrm{~min}$ at room temperature in the dark, all the samples were analyzed within $1 \mathrm{~h}$ with a BD flow cytometry system with FACSDiva software (BD Biosciences, Franklin Lakes, NJ, USA). The data were analyzed using FlowJo v5.7.2 software (BD Biosciences).

Wound healing assay. The cells were transfected and cultured to near $(>80 \%)$ confluency in 24 -well dishes. Then, an artificial homogeneous wound was created onto the monolayer with a sterile pipette tip, and cultured in RPMI-1640 medium containing $10 \%$ FBS for $24 \mathrm{~h}$. To visualize migrating cells, wound closure was measured by photographing at five selected random fields at the time of wounding (time $0 \mathrm{~h}$ ) and at $24 \mathrm{~h}$ after wounding under a light microscope (Olympus, Tokyo, Japan).

Transwell invasion assay. Cell invasion was performed using Transwell chamber assay $(8.0-\mu \mathrm{m}$ pore size; Corning Inc., Corning, NY, USA). Briefly, the $2 \times 10^{4}$ transfected cells were seeded onto the upper chamber coated with Matrigel (BD Biosciences, Bedford, MA, USA) in serum-free medium, while the lower chamber was added with $20 \%$ FBS $(600 \mu \mathrm{l})$. After the cells were incubated for $48 \mathrm{~h}$ at $37^{\circ} \mathrm{C}$ with $5 \% \mathrm{CO}_{2}$, the upper chamber was removed and the cells that had migrated to the lower chamber through the membrane were fixed in $90 \%$ alcohol and stained with $0.1 \%$ crystal violet for $5 \mathrm{~min}$, then, photographed under a light microscope (magnification, x200; Olympus). The number of invaded cells was counted at five randomly selected fields.

Luciferase reporter assay. Regions of the 3' untranslated region (3'UTR) for STAT3 containing the miR-124 binding sites was amplified from human genomic DNA, and inserted into the pGL3-control vector (Ambion, Austin, TX, USA) at the NheI and XhoI restriction sites, named as: Wt-STAT3-3'UTR. Mutant STAT3 containing four-point mutations in the putative miR-124 seed recognition motif were generated using overlapping PCR, and then inserted into the pGL3-control vector (Ambion) at the NheI and XhoI restriction sites, named as: Mut-STAT3-3'UTR. For the luciferase assay, RB cells were seeded onto 12 -well plates at $\sim 70 \%$ confluency and co-transfected with $50 \mathrm{ng}$ of plasmid DNA and $50 \mathrm{nM}$ miR-124 or miR-NC using Lipofectamine 2000 according to the manufacturer's protocol. Measurements of firefly and Renilla luciferase activity were performed $48 \mathrm{~h}$ after transfection using the Dual-Luciferase Reporter Assay System (Promega, Madison, WI, USA). Renilla luciferase was used for normalization. 

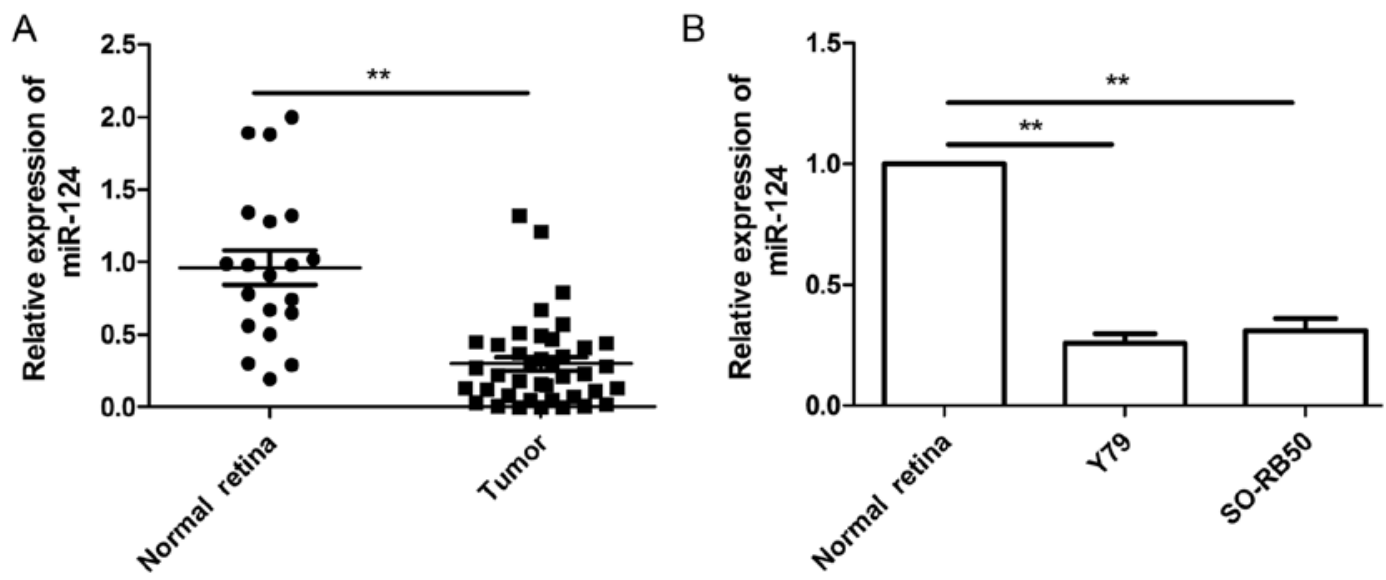

Figure 1. miR-124 expression is downregulated in primary retinoblastoma (RB) tissues and cell lines. (A) The expression levels of miR-124 were determined in 20 normal retina samples and $40 \mathrm{RB}$ tumor samples by quantitative real-time polymerase chain reaction (qRT-PCR). (B) The expression levels of miR-124 were determined in two RB cell lines (Y79 and SO-RB50); ${ }^{* *} \mathrm{P}<0.01$ compared with the normal retina samples.

Western blot analysis. Total proteins of the cell lines and tissues were extracted using a RIPA buffer with $0.5 \%$ sodium dodecyl sulfate (SDS) in the presence of a proteinase inhibitor cocktail (Complete Mini; Roche Diagnostics, Basel, Switzerland). The concentration of protein was quantified using a Bradford assay protein assay kit (Beyotime Biotech, Shanghai, China). Equal amounts of protein $(20 \mu \mathrm{g})$ were separated using $10 \%$ SDS-polyacrylamide gels (SDS-PAGE) and transferred to polyvinylidene fluoride (PVDF) membranes (Millipore, Bedford, MA, USA) for $1 \mathrm{~h}$ at $100 \mathrm{~V}$ at $4^{\circ} \mathrm{C}$. The membranes were blocked with $5 \%$ non-fat milk/Tris-buffered saline with Tween-20 (TBST) for $1 \mathrm{~h}$ at room temperature, and then, incubated at room temperature with primary antibodies against STAT3 $(1: 1,000)$ and GAPDH $(1: 3,000)$ (both from Santa Cruz Biotechnology, Inc., Santa Cruz, CA, USA). GAPDH was used as control. Membranes were incubated with corresponding horseradish peroxidase (HRP)-conjugated secondary antibody (1:0000; Santa Cruz Biotechnology, Inc.) for $2 \mathrm{~h}$ at room temperature. Proteins were detected using an enhanced chemiluminescence luminol-based reagent, and visualized on X-ray film under an ECL detection system (both from Thermo Fisher Scientific, Inc., Waltham, MA, USA).

Statistical analysis. Data from at least three independent experiments are expressed as mean \pm standard deviation (SD). Statistical analysis was performed using IBM SPSS 19.0 statistical software (version 19.0; SPSS, Inc., Chicago, IL, USA). Statistical analysis was performed using Student's t-test or one-way ANOVA. Statistical significance was considered to indicate a statistically significant result at a P-value of $<0.05$.

\section{Results}

miR-124 expression is downregulated in primary RB tissues and cell lines. Quantitative real-time polymerase chain reaction (qRT-PCR) was performed to examine the expression levels of miR-124 in 20 normal retina and $40 \mathrm{RB}$ tumor samples, and normalized against endogenous U6 controls. As shown in Fig. 1A, the expression level of miR-124 in RB tissues was significantly downregulated when compared to the normal retina tissues. Next, we evaluated the expression of miR-204 in two human RB cell lines (Y79 and SO-RB50). In comparison to normal retina tissues, miR-204 was downregulated in two cell lines (Fig. 1B). These data suggested that miR-124 may play crucial roles in the RB process.

miR-124 inhibits cell proliferation and induces cell apoptosis in RB cells. To assess the role of miR-124 in the growth of $\mathrm{RB}$, miR-124 mimic and miR-NC were transiently transfected into human RB cell lines (Y79 and SO-RB50), qRT-PCR was used to confirm miR-124 overexpression in two RB cells (Fig. 2A). The MTT assay was performed to investigate the effect of miR-124 in RB cell proliferation. The results showed that restoration of miR-124 significantly inhibited the proliferation of Y79 and SO-RB50 cells (Fig. 2B). In addition, cell apoptosis assay was performed in RB cells transfected with miR-124 or miR-NC to investigate the effect of miR-124 on apoptosis in RB cells. Our results showed that restoration of miR-124 significantly increased the apoptosis rate in Y79 cells and SO-RB50 cells (Fig. 2C).

miR-124 inhibits cell proliferation in RB cells. To examine the effect of miR-124 on cell metastasis, cell migration and invasion were determined in RB cells transfected with miR-124 mimic or miR-NC by wound healing and Transwell invasion assays, respectively. It was found that restoration of miR-124 significantly inhibited migration (Fig. 3A) and invasion (Fig. 3B) capacities in Y79 and SO-RB50 cells.

STAT3 is a direct target of miR-124 in RB cells. It has been confirmed that STAT3 is the direct target of miR-124 in many cancer cells (18-21). Considering the tissue-specific and developmental stage-specific manner of miRNAs, we wondered whether STAT3 also is a direct target of miR-124 expression in RB cell lines, thus, luciferase activity was assessed in RB cells co-transfected with miR-124 or miR-NC and Wt-STAT3-3'UTR or Mut-STAT3-3'UTR. As shown in Fig. 4A and B, miR-124 significantly inhibited the luciferase activity of the Wt-STAT33'UTR, but not that of the Mut-STAT3-3'UTR in Y79 and SO-RB50 cells. To directly assess the effect of miR-124 on 
A

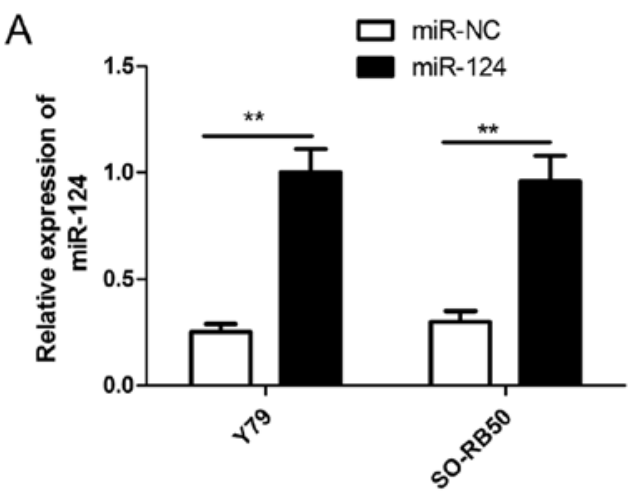

C

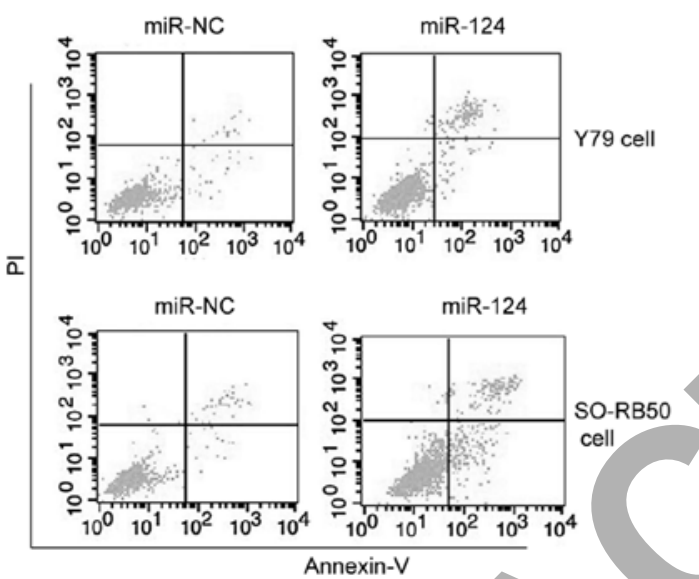

B
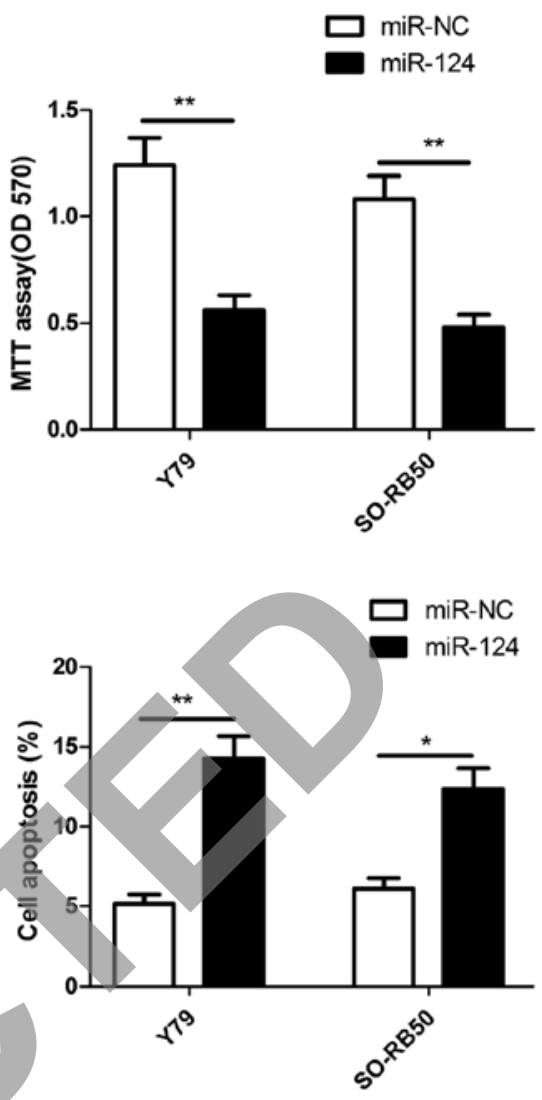

Figure 2. miR-124 inhibits cell proliferation and induces cell apoptosis in RB cells. (A) The expression levels of miR-124 were determined by qRT-PCR in RB cells transfected with miR-124 or miR-NC by qRT-PCR. (B) Cell proliferation was determined by MTT assay in RB cells transfected with miR-124 or miR-NC. (C) Cell apoptosis was detected by flow cytometric assay in RB cells transfected with miR-124 or miR-NC; ${ }^{* *} \mathrm{P}<0.01$ compared with the miR-NC.
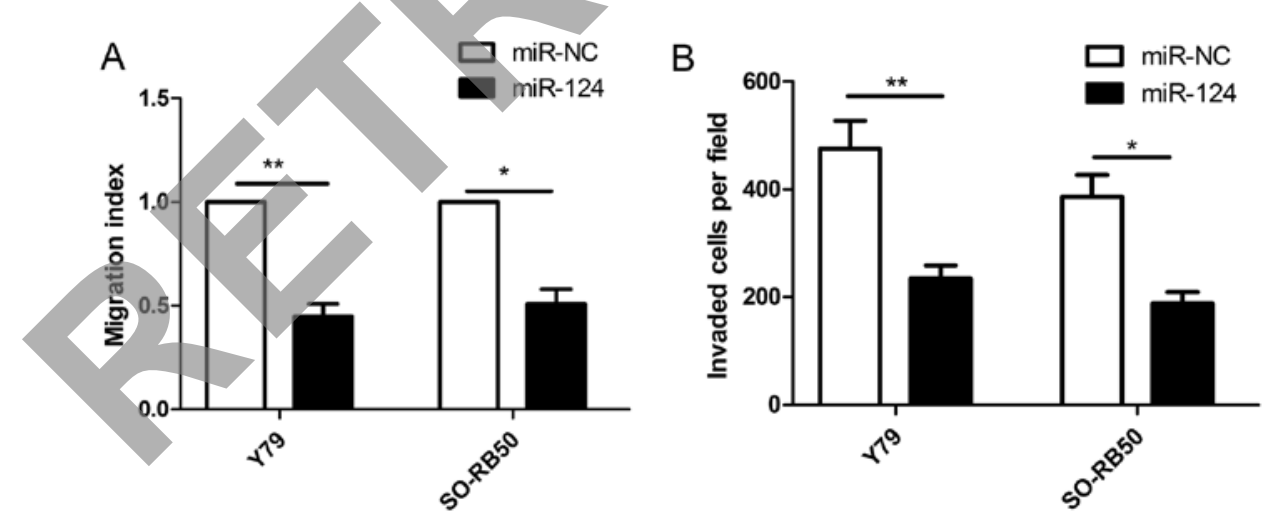

Figure 3. miR-124 inhibits cell migration and invasion in RB cells. (A) Cell migration was determined by wound healing assay in RB cells transfected with miR-124 or miR-NC. (B) Cell invasion was detected by Transwell invasion assay in RB cells transfected with miR-124 or miR-NC; ${ }^{* *} \mathrm{P}<0.01$ compared with the miR-NC.

expression, we transfected miR-124 or miR-NC into RB cells, and found that overexpression of miR-124 reduced the STAT3 mRNA level (Fig. 4C) and protein expression (Fig. 4D) in Y79 and SO-RB50 cells. These results demonstrated that STAT3 is a direct target of miR-124 in RB cells.

STAT3 expression is inversely correlated with miR-124 expression in $R B$ tissues. The above results proved that STAT3 is the direct target of miR-124 in RB cells, we investigated therefore STAT3 mRNA expression in RB tissues and normal retina samples by qRT-PCR. Our results showed that STAT3 expression on mRNA level was upregulated in RB tissues compared to normal retina samples (Fig. 5A). In addition, a statistically significant inverse correlation was revealed by Spearman's correlation analysis between miR-124 and STAT3 mRNA levels in RB tissues $(r=-0.638$; $\mathrm{P}<0.001$; Fig. 5B).

STAT3 reverses the inhibitory effect of miR-124 on cell proliferation, migration and invasion in $R B$ cells. To investigate whether miR-124 mediates its inhibition effects in RB cells through STAT3, we increased the miR-124 level in human 

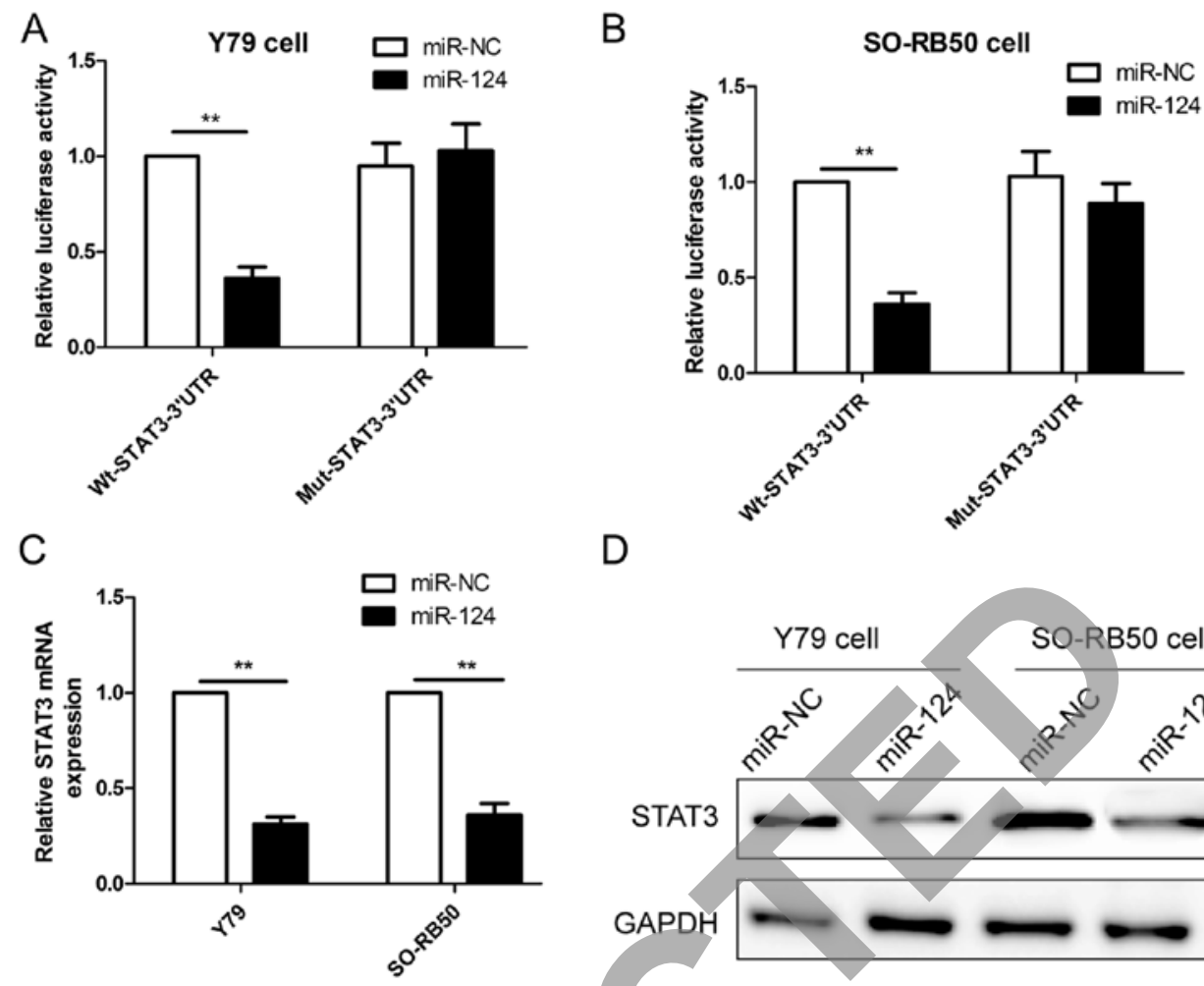

$\mathrm{D}$

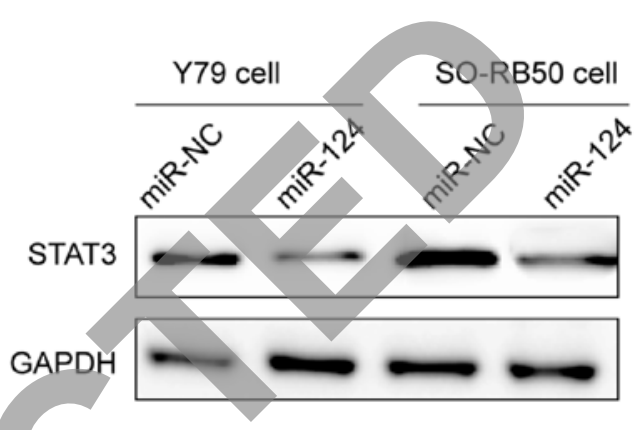

Figure 4. STAT3 is a direct target of miR-124 in RB cells. (A and B) Luciferase reporter assay was performed in (A) Y79 and (B) SO-RB50 cells transfected with the Wt or Mut-STAT3-3'UTR reporter plasmid and miR-124 mimic or miR-NC. Wt, wild-type; Mut, mutant-type. (C) The STAT3 mRNA level was determined by qRT-PCR in RB cells transfected with miR-124 or miR-NC. (D) The STAT3 protein expression was determined by western blotting in RB cells transfected with miR-124 or miR-NC. GAPDH was used as an internal control; ${ }^{*} \mathrm{P}<0.05,{ }^{* *} \mathrm{P}<0.01$ compared with the miR-NC.
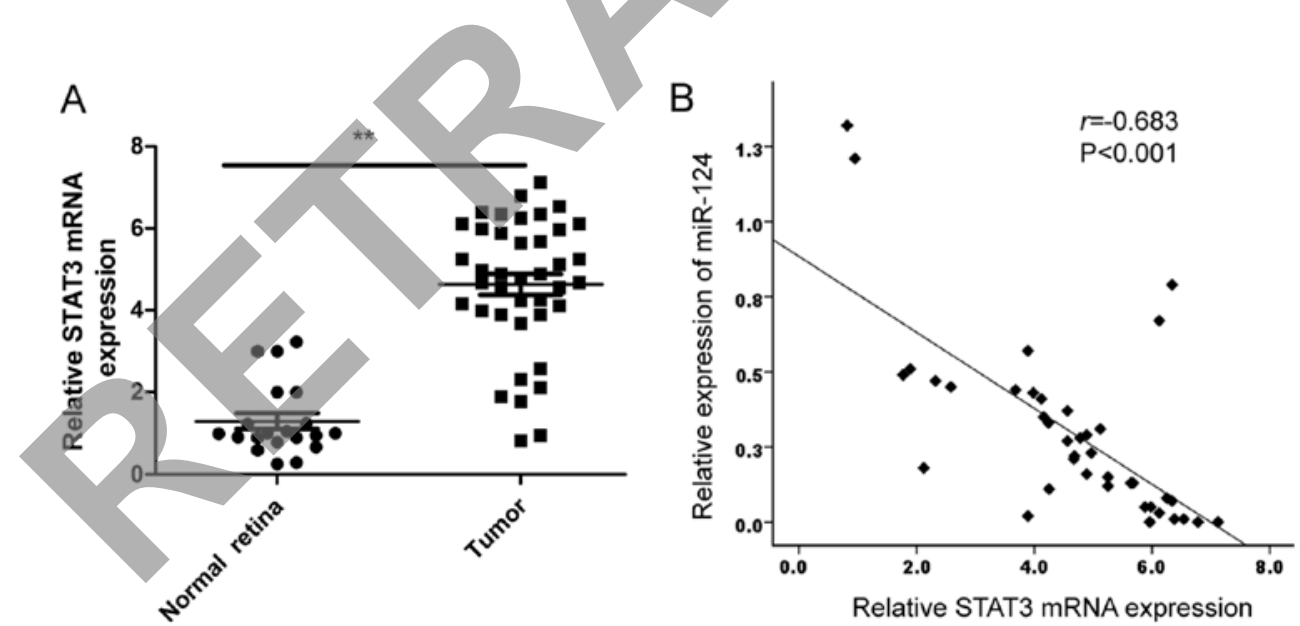

Figure 5. STAT3 expression is inversely correlated with miR-124 expression in RB tissues. (A) The STAT3 mRNA level was determined in 20 normal retina and 40 RB tumor samples by qRT-PCR; ${ }^{* *} \mathrm{P}<0.01$ compared to normal retina samples. (B) The reverse relationship between STAT3 and miR-124 expression was explored by Spearman's correlation in RB tissues.

RB cells using a miR-124 mimic and rescued the expression of STAT3 (using the STAT3 overexpression vector without its 3 'UTR) through the transfection of the miR-124 mimic in RB cells. The result of western blot analysis showed that the miR-124 mimic obviously inhibited STAT3 protein expression compared with the miR-NC in Y79 cells and SO-RB50 cells, while the overexpression of STAT3 abolished the inhibition caused by the miR-124 mimic (Fig. 6A). Notably, overexpression of STAT3 rescues the suppressive effects on proliferation (Fig. 6B), migration (Fig. 6C) and invasion (Fig. 6D) in RB cells caused by miR-124 expression in
RB cells. These results suggested that miR-124 exerted its suppressive effecs in RB cells, at least in part, by regulating STAT3.

\section{Discussion}

A large number of microRNAs (miRNAs) has been identified to be involved in occurrence and development of retinoblastoma $(\mathrm{RB})$ through regulating and inhibiting the expression of their target gene, and functioned as oncogene or tumor suppressor by regulating proliferation, cell cycle, 
A
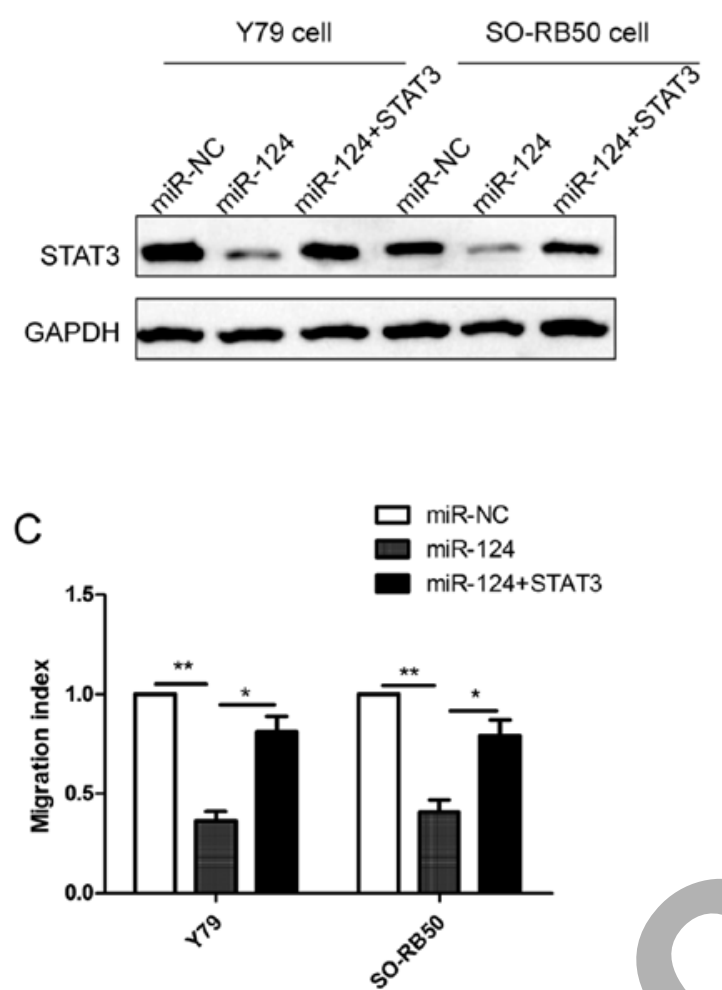

B
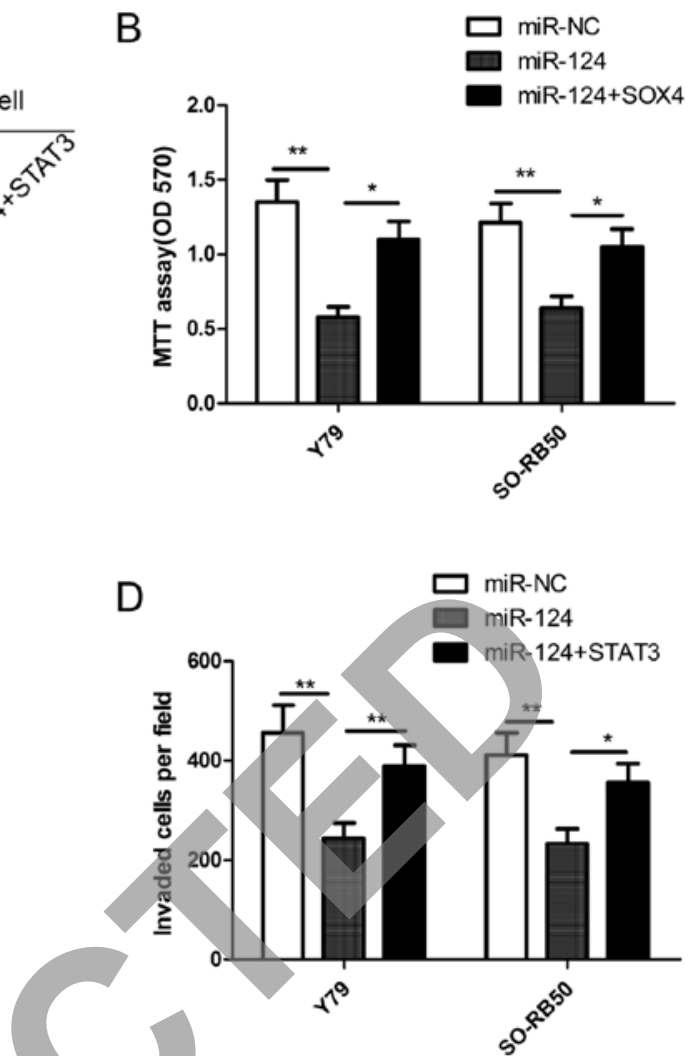

Figure 6. STAT3 reverses the inhibitory effect of miR-124 on cell proliferation, migration and invasion in RB cells. (A). STAT3 protein was measured by western blotting in RB cells transfected with miR-124 with/without STAT3 overexpression plasmid. GAPDH was used as an internal control. (B) Cell proliferation was determined by MTT assay in RB cells $72 \mathrm{~h}$ after transfection with miR-124 with/without STAT3 overexpression plasmid. (C) Cell migration was determined by wound healing assay in RB cells $24 \mathrm{~h}$ after transfection with miR-124 with/without STAT3 overexpression plasmid. (D) Cell invasion was determined by Transwell invasion assay in RB cells $48 \mathrm{~h}$ after transfection with miR-124 with/without STAT3 overexpression plasmid; " $\mathrm{P}<0.05$, ${ }^{* *} \mathrm{P}<0.01$ compared with the miR-124.

apoptosis, invasion and migration of $\mathrm{RB}(10,11)$. Wu et al reported that miR-204 was frequently downregulated in RB tissues and cell lines, and that enforced expression of miR-204 inhibited the RB cell proliferation and invasion by targeting cyclin D2 and MMP-9 (22). Sun et al demonstrated that miR-145 suppressed RB cell proliferation, migration and invasion by repressing a disintegrin and metalloproteinases 9 (ADAM9) (23). Wang et al found that miR-183 suppressed proliferation, migration and invasion of $\mathrm{RB}$ cells by downregulation of low-density lipoprotein receptor-related protein 6 (LRP6) (24). In the present study, our results showed that miR-124 expression is downregulated in human RB tissues and RB cell lines compared with normal retinal tissue. Our results also demonstrated that restoration of miR-124 inhibited RB cell proliferation, migration and invasion, and induced cell apoptosis by targeting STAT3. These results support the conclusion that miR-124 plays a crucial role in RB development.

miR-124, located in $8 \mathrm{q} 12.3$, is frequently found to be downregulated in multiple human malignancies, such as prostate cancer (12), glioma (13), lung adenocarcinoma (14), breast (15), gastric (16), colorectal (17) and bladder cancer (25), hepatocellular carcinoma (20) and ovarian cancer (26). miR-124 exerted a tumor suppressive role in various cancer cells by negative regulation of cell proliferation, apoptosis, migration and invasion through repressing multiple target genes, such as
PACE4 (12), SOX9 (14), CD4 (25), STAT3 (20), SphK1 (26) and PIK3CA (27). However, at present, there is no published study regarding the biological functions of miR-124 in RB. In the present study, we found that miR-124 expression was downregulated in RB tissues and cell lines, and that miR-124 suppressed cell proliferation, migration and invasion of $\mathrm{RB}$ cells. These results suggested that miR-124 functioned as a tumor suppressor in RB cells.

STAT3, a member of the signal transducer and activator of transcription (STAT family), has been shown to play crucial roles in cell cycle progression, apoptosis, cellular transformation and proliferation by regulating the expression of multiple target genes such as cyclin D1, c-Myc, survivin, Bcl-xL, Bcl-2, Mcl-1, VEGF and MMP-2 and MMP-9 (28-34). It was found that downregulation of STAT3 using RNA interference targeting STAT3, and small molecule inhibitors suppressed tumor cell proliferation and invasion, induced apoptosis in vitro, and delayed tumor growth in animal models of various types of cancer (32-34). Recently, a study showed that STAT3 expression was increased in RB tissues from human patients compared to normal retinal tissues, and that inhibition of STAT3 in RB cells with targeted siRNAs resulted in impaired proliferation and downregulation of target genes in vitro, and suppressed formation of orthotopic tumors in vivo (35), suggesting STAT3 is an oncogene in RB. Although STAT3 has been reported to be a target of miR-124 in several types 
of cancers, such as esophageal cancer (18), glioblastoma (19), hepatocellular carcinoma (20) and non-small cell lung cancer (21), however, the interaction between miR-124 and STAT3 has not been experimentally validated in RB. In the present study, we confirmed that STAT3 was a direct target of miR-124, and that miR-124 overexpression significantly reduced the levels of both STAT3 protein and mRNA in RB cells. We also demonstrated that miR-124 expression levels negatively correlated with STAT3 mRNA levels in human RB tissues specimens. STAT3 overexpression rescued the suppressive effect of miR-124-mediated RB cell proliferation, migration and invasion. These results displayed evidence of miR-124-mediated suppression role in RB cells, at least in part by targeting STAT3.

In summary, to the best of our knowledge, the present study is the first to provide evidence that the expression of miR-124 is downregulated in RB tissues and cell lines; and that restoration of miR-124 inhibited proliferation, migration and invasion, and induced cell apoptosis in RB cells, at least in part, by targeting STAT3. These findings suggested that miR-124 functioned as tumor-suppressor, and may become a novel molecular therapeutic target for the treatment of RB.

\section{References}

1. Shields CL and Shields JA: Retinoblastoma management: Advances in enucleation, intravenous chemoreduction, and intra-arterial chemotherapy. Curr Opin Ophthalmol 21: 203-212, 2010.

2. Jabbour P, Chalouhi N, Tjoumakaris S, Gonzalez LF, Dumont AS, Chitale R, Rosenwasser R, Bianciotto CG and Shields C: Pearls and pitfalls of intraarterial chemotherapy for retinoblastoma. J Neurosurg Pediatr 10: 175-181, 2012.

3. Abramson DH, Marr BP, Brodie S, Dunkel IJ and Gobin PY: Intraarterial chemotherapy for kissing macula tumors in retinoblastoma. Retin Cases Brief Rep 6:209-211, 2012.

4. Meel R, Radhakrishnan V and Bạkhshi S: Current therapy and recent advances in the management of retinoblastoma. Indian $\mathrm{J}$ Med Paediatr Oncol 33: 80-88, 2012.

5. He L and Hannon GJ: MicroRNAs: Small RNAs with a big role in gene regulation. Nat Rev Genet 5: 522-531, 2004.

6. Erhard F, Haas J, Lieber D, Malterer G, Jaskiewicz L, Zavolan M, Dölken L and Zimmer R: Widespread context dependency of microRNA-mediated regulation. Genome Res 24: 906-919, 2014

7. Bartel DP: MicroRNAs: Genomics, biogenesis, mechanism, and function. Cell 116: 281-297, 2004.

8. Zhang B, Pan X, Cobb GP and Anderson TA: microRNAs as oncogenes and tumor suppressors. Dev Biol 302: 1-12, 2007.

9. Calin GA and Croce CM: MicroRNA signatures in human cancers. Nat Rev Cancer 6: 857-866, 2006.

10. Yang Y and Mei Q: miRNA signature identification of retinoblastoma and the correlations between differentially expressed miRNAs during retinoblastoma progression. Mol Vis 21: 1307-1317, 2015

11. Beta M, Venkatesan N, Vasudevan M, Vetrivel U, Khetan V and Krishnakumar S: Identification and insilico analysis of retinoblastoma serum microRNA profile and gene targets towards prediction of novel serum biomarkers. Bioinform Biol Insights 7: 21-34, 2013.

12. Kang S, Zhao Y, Hu K, Xu C, Wang L, Liu J, Yao A, Zhang H and Cao F: miR-124 exhibits antiproliferative and antiaggressive effects on prostate cancer cells through PACE4 pathway. Prostate 74: 1095-1106, 2014

13. Xia H, Cheung WK, Ng SS, Jiang X, Jiang S, Sze J, Leung GK, Lu G, Chan DT, Bian XW, et al: Loss of brain-enriched miR-124 microRNA enhances stem-like traits and invasiveness of glioma cells. J Biol Chem 287: 9962-9971, 2012.

14. Wang X, Liu Y, Liu X, Yang J, Teng G, Zhang L and Zhou C: miR-124 inhibits cell proliferation, migration and invasion by directly targeting SOX9 in lung adenocarcinoma. Oncol Rep 35: $3115-3121,2016$
15. Feng T, Shao F, Wu Q, Zhang X, Xu D, Qian K, Xie Y, Wang S, $\mathrm{Xu}$ N, Wang Y, et al: miR-124 downregulation leads to breast cancer progression via LncRNA-MALAT1 regulation and CDK4/E2F1 signal activation. Oncotarget 7: 16205-16216, 2016.

16. Jiang L, Lin T, Xu C, Hu S, Pan Y and Jin R: miR-124 interacts with the Notch1 signalling pathway and has therapeutic potential against gastric cancer. J Cell Mol Med 20: 313-322, 2016.

17. Xi ZW, Xin SY, Zhou LQ, Yuan HX, Wang Q and Chen KX: Downregulation of rho-associated protein kinase 1 by miR-124 in colorectal cancer. World J Gastroenterol 21: 5454-5464, 2015.

18. Cheng Y, Li Y, Nian Y, Liu D, Dai F and Zhang J: STAT3 is involved in miR-124-mediated suppressive effects on esophageal cancer cells. BMC Cancer 15: 306, 2015.

19. Li W, Huang H, Su J, Ji X, Zhang X, Zhang Z and Wang H: miR-124 acts as a tumor suppressor in glioblastoma via the inhibition of signal transducer and activator of transcription 3. Mol Neurobiol: Mar 18, 2016 (Epub ahead of print).

20. Lu Y, Yue X, Cui Y, Zhang J and Wang K: MicroRNA-124 suppresses growth of human hepatocellular carcinoma by targeting STAT3. Biochem Biophys Res Commun 441: 873-879, 2013.

21. Li X, Yu Z, Li Y, Liu S, Gao C, Hou X, Yao R and Cui L: The tumor suppressor miR-124 inhibits cell proliferation by targeting STAT3 and functions as a prognostic marker for postoperative NSCLC patients. Int J Oncol 46: 798-808, 2015.

22. Wu X, Zeng Y, Wu S, Zhong J, Wang Y and Xu J: MiR-204, down-regulated in retinoblastoma, regulates proliferation and invasion of human retinoblastoma cells by targeting CyclinD2 and MMP-9. FEBS Lett 589: 645-650, 2015.

23. Sun Z, Zhang A, Jiang T, Du Z, Che C and Wang F: MiR-145 suppressed human retinoblastoma cell proliferation and invasion by targeting ADAM19. Int J Clin Exp Pathol 8: 14521-14527, 2015.

24. Wang J, Wang X, Li Z, Liu H and Teng Y: MicroRNA-183 suppresses retinoblastoma cell growth, invasion and migration by targeting LRP6. FEBS J 281: 1355-1365, 2014.

25. Zhang T, Wang J, Zhai X, Li H, Li C and Chang J: MiR-124 retards bladder cancer growth by directly targeting CDK4. Acta Biochim Biophys Sin 46: 1072-1079, 2014.

26. Zhang H, Wang Q, Zhao Q and Di W: MiR-124 inhibits the migration and invasion of ovarian cancer cells by targeting SphK1. J Ovarian Res 6: 84, 2013.

27. Lang Q and Ling C: MiR-124 suppresses cell proliferation in hepatocellular carcinoma by targeting PIK3CA. Biochem Biophys Res Commun 426: 247-252, 2012.

28. Turkson J: STAT proteins as novel targets for cancer drug discovery. Expert Opin Ther Targets 8: 409-422, 2004.

29. Masuda M, Suzui M, Yasumatu R, Nakashima T, Kuratomi Y, Azuma K, Tomita K, Komiyama S and Weinstein IB: Constitutive activation of signal transducers and activators of transcription 3 correlates with cyclin D1 overexpression and may provide a novel prognostic marker in head and neck squamous cell carcinoma. Cancer Res 62: 3351-3355, 2002.

30. Wei D, Le X, Zheng L, Wang L, Frey JA, Gao AC, Peng Z, Huang S, Xiong HQ, Abbruzzese JL, et al: Stat 3 activation regulates the expression of vascular endothelial growth factor and human pancreatic cancer angiogenesis and metastasis. Oncogene 22: 319-329, 2003.

31. Xie TX, Wei D, Liu M, Gao AC, Ali-Osman F, Sawaya R and Huang S: Stat3 activation regulates the expression of matrix metalloproteinase-2 and tumor invasion and metastasis. Oncogene 23: 3550-3560, 2004.

32. Furtek SL, Backos DS, Matheson CJ and Reigan P: Strategies and approaches of targeting STAT3 for cancer treatment. ACS Chem Biol 11: 308-318, 2016.

33. Suh YA, Jo SY, Lee HY and Lee C: Inhibition of IL-6/STAT3 axis and targeting Axl and Tyro3 receptor tyrosine kinases by apigenin circumvent taxol resistance in ovarian cancer cells. Int J Oncol 46: 1405-1411, 2015.

34. Chai EZ, Shanmugam MK, Arfuso F, Dharmarajan A, Wang C, Kumar AP, Samy RP, Lim LH, Wang L, Goh BC, et al: Targeting transcription factor STAT3 for cancer prevention and therapy. Pharmacol Ther 162: 86-97, 2016.

35. Jo DH, Kim JH, Cho CS, Cho YL, Jun HO, Yu YS, min JK and Kim JH: STAT3 inhibition suppresses proliferation of retinoblastoma through down-regulation of positive feedback loop of STAT3/miR-17-92 clusters. Oncotarget 5: 11513-11525, 2014. 